\title{
A Pilot Trial of the Alpha-1 Adrenergic Antagonist, Prazosin, for Alcohol Dependence
}

\author{
Tracy L. Simpson, Andrew J. Saxon, Charles W. Meredith, Carol A. Malte, Brittney McBride, \\ Laura C. Ferguson, Christopher A. Gross, Kim L. Hart, and Murray Raskind
}

\begin{abstract}
Background: Current medications for alcohol dependence (AD) show only modest efficacy. None target brain noradrenergic pathways. Theory and preclinical evidence suggest that noradrenergic circuits may be involved in alcohol reinforcement and relapse. We therefore tested the $\alpha-1$ adrenergic receptor antagonist, prazosin, as a pharmacotherapy for AD.

Methods: We randomized 24 participants with AD but without posttraumatic stress disorder to receive either prazosin or placebo in a 6-week, double-blind pilot study. Medication was titrated to a target dose of $4 \mathrm{mg}$ QAM, $4 \mathrm{mg}$ QPM, and $8 \mathrm{mg}$ QHS by the end of week 2. Participants received 5 medical management treatment sessions. Participants were reminded 3 times each day via a text pager to take medications and to call a telephone monitoring system once daily to provide self-reports of alcohol consumption and craving, the primary outcome measures. Results were analyzed using mixed linear regression adjusted for drinking days per week at baseline and week number.

Results: Twenty of the $24(83 \%)$ subjects completed. Among the completers, the prazosin group reported fewer drinking days per week than the placebo group during the final 3 weeks of the study. Since only 1 woman was randomized to placebo and only three women completed the trial, the following results focus on the 17 male completers. The prazosin group reported fewer drinking days per week and fewer drinks per week during the final 3 weeks of the study; average total number of drinking days for the placebo group 5.7 (SEM 1.9) versus 0.9 (SEM 0.5) for the prazosin group, and average total number of drinks 20.8 (SEM 6.5) for the placebo group versus 2.6 (SEM 1.3) for the prazosin group. Rates of adverse events were equivalent across conditions.

Conclusions: Prazosin holds promise as a pharmacologic treatment for AD and deserves further evaluation in a larger controlled trial.
\end{abstract}

Key Words: Noradrenergic, Prazosin, Alcohol Dependence, Human Clinical Trial.

\section{A}

LCOHOL DEPENDENCE (AD) is a biologically, genetically based disease (Heath et al., 1997; Kendler et al., 1994), yet the majority of clinically accepted treatments are behaviorally or psychosocially based (Anton and Swift, 2003; Swift, 1999). In light of the high relapse rates associated with these treatments, which range from $40 \%$ to $70 \%$ within the first 12 months after treatment (Finney et al., 1996), research is needed to develop more effective biological treatments.

Disulfiram is the oldest FDA approved medication for the treatment of $\mathrm{AD}$ and shows modest efficacy in reducing

From the Center of Excellence in Substance Abuse Treatment and Education (TLS, AJS, CAM, BM); Mental Illness Research Education and Clinical Center (TLS, CWM, LCF, CAG, KLH, MR), VA Puget Sound Health Care System; and Department of Psychiatry and Behavioral Sciences (TLS, AJS, CWM, LCF, CAG, MR), University of Washington, Seattle, Washington.

Received for publication May 20, 2008; accepted August 6, 2008.

Reprint requests: Tracy L. Simpson, PhD, 1660 S. Columbian Way (116-ATC-S), Seattle, WA 98108; Fax: 206-764-2293; E-mail: Tracy.Simpson@va.gov.

Copyright (C) 2008 by the Research Society on Alcoholism.

DOI: 10.1111/j.1530-0277.2008.00807.x drinking frequency but does not improve abstinence rates (Fuller and Roth, 1979; Fuller et al., 1983, 1986). Its use is limited by its risk of significant hepatotoxicity, the need for regular monitoring of liver function tests, and its contraindication in ischemic heart disease (Swift, 1999). It also has only limited efficacy without directly supervised administration (Chick et al., 1992).

More recently, much attention has focused on the glutamatergic (Nagy, 2004; Woodward et al., 2006), GABAergic (Koob, 2004; Krystal et al., 2006), dopaminergic (Gonzales et al., 2004; Tupala and Tiihonen, 2004), and endogenous opioid systems in AD (Gianoulakis, 2004; Oswald and Wand, 2004). Medications that modulate these systems, naltrexone and acamprosate, have received FDA approval for treating AD. Naltrexone originally showed some promise, but its tolerability is limited by nausea, headache, and sedation (Garbutt et al., 1999; Swift, 1999). A large, well-conducted multicenter trial failed to show a main effect of naltrexone versus placebo (Anton et al., 2006). A study of long acting injectable naltrexone found that it slightly reduced heavy drinking days in men but not in women (Garbutt et al., 2005). A recent meta-analysis of the efficacy of acamprosate indicated that its efficacy varied widely across studies, although with all data included, it slightly increased the odds 
of abstinence compared to placebo (Mann et al., 2004). However, in a large multisite trial not included in the meta-analysis noted above, acamprosate was found inefficacious (Anton et al., 2006; Mason et al., 2006). Thus, both naltrexone and acamprosate have demonstrated at best modest efficacy for AD.

The anti-convulsant topiramate has recently been shown to have efficacy in $\mathrm{AD}$ in a multisite trial, although a 5-week titration period was required, and nearly 6 times as many subjects in the topiramate group dropped out of the study due to adverse effects compared to those in the placebo group (Johnson et al., 2007).

None of the aforementioned medications directly target noradrenergic (NE) brain systems. Recent advances in the understanding of the neurobiology of substance dependence and relapse offer preclinical evidence that NE systems have intimate involvement in brain processes relevant to $\mathrm{AD}$, such as arousal (Aston-Jones and Cohen, 2005), reinforcement (Ventura et al., 2005), and stress responsivity (Koob, 1999). However, virtually no work to date has attempted to translate this knowledge into clinical interventions, though earlier work by Mason et al. (Mason and Kocsis, 1991; Mason et al., 1996) on the norepinephrine reuptake inhibitor, desipramine, among depressed and nondepressed alcohol dependent individuals showed that desipramine prolonged the time to relapse. When depressed subjects were removed from the analysis, there was not a significant difference in time to relapse among placebo and desipramine treated subjects.

Following extensive review of a wide range of experimental and clinical results, Begleiter and Porjesz concluded that hyperexcitability is the key feature of the most parsimonious model of predisposition to develop AD (Begleiter and Porjesz, 1999). Hyperexcitability is associated with increased adrenergic activation. Brain noradrenergic systems and peripheral sympathetic regulation are functionally integrated (Tjurmina et al., 1999), and research with recently abstinent alcohol dependent individuals demonstrates that they have elevated plasma levels of epinephrine (Ehrenreich et al., 1997) and norepinephrine (Patkar et al., 2003, 2004), suggesting central adrenergic overdrive plays an important role in AD.

Enhanced acoustic startle response serves as a proxy for arousal and hyperexcitability. Brain $\alpha_{1}$ adrenergic mechanisms mediate enhanced acoustic startle response (Stevens et al., 1994), which is characteristic of abstinent alcoholics (Krystal et al., 1997), persons with posttraumatic stress disorder (PTSD) (Orr et al., 1995), selectively-bred alcohol preferring P rats (Chester et al., 2004), and outbred rats experiencing prolonged abstinence following long-term chronic daily ethanol consumption and withdrawal (Rasmussen et al., 2005). Emerging evidence also shows that noradrenergic systems modulate midbrain dopaminergic neurons which in turn have key roles in the reinforcing and locomotor activating responses to drugs of abuse, including ethanol. These findings, along with the earlier work by Mason and her colleagues on desipramine for AD (Mason and Kocsis, 1991; Mason et al., 1996), suggest that an $\alpha_{1}$ antagonist may have potential in interrupting a key neurobiological system's contribution to AD.

The work reported here examines the novel, promising strategy of treating AD by reducing central adrenergic activity via blockade of noradrenaline binding to postsynaptic $\alpha_{1}$ receptors with the nonselective, $\alpha_{1}$ antagonist, prazosin. Prazosin was introduced in 1973 as "Minipress" by Pfizer Pharmaceuticals as an antihypertensive drug. An inexpensive generic drug for many years, prazosin has been used chronically by millions of persons for hypertension and for urinary symptoms caused by later life benign prostatic hypertrophy (Hieble and Ruffolo, 1996; Lund-Johansen et al., 1993). Prazosin is the most lipid soluble $\alpha_{1}$ antagonist (Westfall and Westfall, 2006) and the only clinically available $\alpha_{1}$ antagonist demonstrated to be active at central nervous system sites when administered peripherally (Darracq et al., 1998; Menkes et al., 1981). Our interest in prazosin initially derived from the clinical observation that patients with co-occurring PTSD and $\mathrm{AD}$ often reported substantially reduced and even complete cessation of alcohol consumption during prazosin treatment (M.A. Raskind, personal communication, August 1, 2004).

Subsequently, Rasmussen and colleagues tested the hypothesis that prazosin would be useful in reducing both alcohol-induced reward and stress induced relapse in two animal models. In the first preclinical study, they demonstrated that prazosin treatment decreased operant ethanol selfadministration by Wistar rats during acute ethanol withdrawal (Walker et al., 2006). In the second, they demonstrated that both acute and chronic prazosin treatment decreased ethanol consumption by alcohol preferring $\mathrm{P}$ rats (Rasmussen et al., 2008).

Based on these promising preclinical results, we hypothesized that prazosin would be an effective treatment for AD because it has the potential to antagonize the central postsynaptic $\alpha_{1}$ activity involved in drug and stress-induced reinstatement of drug-seeking for substances of abuse, including alcohol. In addition to testing whether prazosin is an effective pharmacological agent in reducing drinking among AD individuals, we were also interested in evaluating whether reduced craving is a potential mechanism of action. Based on the findings explicated above, prazosin should at least modulate or reduce craving.

In order to test the hypothesis that prazosin will be effective in reducing drinking and to begin evaluating the possible impact of prazosin on craving, we completed a 6-week pilot clinical medication trial of prazosin in individuals seeking treatment for AD. The 6-week timeframe was judged to be sufficient for this pilot endeavor because it was largely geared towards establishing whether there was a positive signal for prazosin, and determining whether the medication and intensive study protocol were acceptable to study participants. Daily interactive voice response (IVR) telephone monitoring was used to assess alcohol use, craving, craving resistance, and other mood states in this study, and complements traditional retrospective outcome assessments. Daily IVR has 
successfully been used in other clinical AD trials (Kranzler et al., 2004) and has not been found to be associated with undue measurement reactivity (Simpson et al., 2005).

\section{METHODS}

\section{Participants}

Nineteen men and 5 women, aged 18 to $69(\mathrm{M}$ age $=45.5 \pm 8.0)$, who met DSM-IV criteria for AD in the past year with last use of alcohol in the last month and were currently seeking treatment for $\mathrm{AD}$ were enrolled in the study. The majority of the sample was Caucasian $(83 \%)$ and living in their own homes $(87.5 \%)$. One-quarter of the sample was currently married and one-third was veterans. The sample reported drinking alcohol on $64.3 \%$ of days during the previous 6 weeks and reported heavy drinking [5 or more Standard Drink Units (SDU's) for men and 4 or more for women] on $46.4 \%$ of these days. On average the sample reported having consumed 192.6 (SD 19.5) SDU's during the 6 weeks prior to the baseline assessment.

An additional 120 individuals inquired about the study but were not included for the following reasons: current PTSD or other psychiatric condition requiring medication other than an anti-depressant $(n=28)$; medical contraindications or already taking prazosin $(n=6)$; not interested once learned what was involved, including not willing to be abstinent $(n=37)$; no drinking past month or no $\mathrm{AD}(n=12)$; currently using opiates $(n=4)$; in other treatment or in controlled living environment $(n=21)$; scheduling difficulties or no reliable way to contact $(n=11)$, and outside of age range $(n=1)$.

All study participants had current DSM-IV AD confirmed by the administration of the DSM-IV Checklist by a physician, nurse practitioner or physician assistant experienced in the diagnosis of $\mathrm{AD}$. Using the criteria in the Structured Clinical Interview for DSM-IV (SCID; First et al., 1995) for delineating mild, moderate, and severe AD where 3 endorsed symptoms is mild dependence, 7 endorsed symptoms is severe, and moderate dependence is 4 through $6,12.5 \%$ of the sample was in the mild range, $54.2 \%$ in the moderate range, and $33.3 \%$ in the severe range. Because of prazosin's demonstrated efficacy in treatment of PTSD symptomatology, we excluded potential participants with PTSD. Potential participants were administered the PTSD Checklist (PCL), a sensitive screening instrument for PTSD (Weathers et al., 1993). If potential subjects scored at or above established cut off scores for a likely diagnosis of PTSD [50 for men (Weathers et al., 1993), 38 for women (Dobie et al., 2002)], they were administered the PTSD section of the SCID by a research psychiatrist experienced in the diagnosis of PTSD. If they were found to have a confirmed diagnosis of PTSD on this instrument, they were excluded. Potential participants were also excluded for psychiatric disorder requiring initiation of medication at time of study entry other than anti-depressants; current diagnosis of opioid dependence or abuse; systolic blood pressure (BP) < $110 \mathrm{~mm} \mathrm{Hg}$; preexisting orthostatic BP; unstable angina, Meniere's disease, narcolepsy, benign positional vertigo, chronic renal or hepatic failure, pancreatitis or insulin-dependent diabetes, or other medical problems requiring immediate attention. Female participants of child-bearing age were not permitted to enroll unless they reported using a birthcontrol method during the study judged by the study clinician to be effective. During their 6 weeks of participation, all subjects were asked to forgo any concurrent treatment for $\mathrm{AD}$ other than Alcoholics Anonymous (AA).

\section{Procedures}

Recruitment and Screening. Human subjects approval was provided by the institutional review board at the University of Washington. Participants were recruited between November 2005 and August 2007 through advertisements in local newspapers, flyers posted at the VA Puget Sound Healthcare System, and announcements made by study staff during orientation group meetings for patients wishing to begin treatment for $\mathrm{AD}$ at our facility's Addiction Treatment Center.

After passing a breathalyzer test to rule out intoxication, participants then signed the informed consent and underwent screening. Screening consisted of a physical examination including weight, height, and vital signs; providing a psychiatric and medical history to a study psychiatrist; PCL (Weathers et al., 1993) DSM-IV checklist for substance dependence and abuse, and for those screening positive for PTSD on the PCL, the SCID PTSD section.

Those found eligible for the study were invited to participate in a baseline assessment either the same day or within the following week. The following measures were administered: Demographic information interview; 6-week Form-90 (Form-42) (Miller and Del Boca, 1994) for use of alcohol and illicit drugs; Pennsylvania Alcohol craving Scale (PACS) (Flannery et al., 1999) and the PACS modified for cocaine and marijuana; laboratory assessment of CBC, electrolytes, liver function panel, urine pregnancy, and urine toxicology screen.

Randomization. Participants who completed the screening process and the baseline assessment were then randomized by our center's research pharmacist to placebo or prazosin and invited back to begin study participation. No participant characteristics were taken into consideration in the randomization. All study personnel and participants remained blinded to each participant's group assignment throughout the course of the study.

Study Visits. During the first 2 weeks of the study, participants completed twice weekly study visits that decreased to weekly through weeks 3 to 6 , during which they had orthostatic vital sign checks and monitoring of adverse events. The study psychiatrist slowed participants' titration if they were experiencing symptomatic orthostatic hypotension or reporting side-effects that they attributed to the study medication and reported to be intolerable; the study psychiatrist had authority to recommend renewal of the prior titration at cessation of participants' reported side-effects. Participants also provided urine toxicology specimens and were asked about medication compliance at each of these visits. They underwent repeat testing of liver function tests at the end of weeks 3 and 6 . In addition, participants repeated the PACS and Form-42 at the end of week 6. At study completion participants were asked whether they thought they had been in the placebo or the prazosin group and to indicate their level of certainty on a visual analog scale.

Remuneration. Participants were remunerated for study visits, lab visits, and for IVR daily monitoring and could earn up to $\$ 191$ for perfect compliance with all aspects of the study.

IVR Daily Monitoring. In order to better track daily fluctuations in alcohol use and craving, as well as medication compliance, we utilized daily IVR symptom monitoring. Participants were paged daily via a text messaging pager reminding them to call a toll-free number to report on their alcohol craving, alcohol use, marijuana craving, marijuana use, cocaine craving, cocaine use, medication compliance, and general emotional well-being over the prior 24 hours. They were also reminded to take their study medication and were paged two additional times later each day to remind them to take the other two doses of study medication.

The IVR protocol was adapted from the work of Searles et al. (2000) and consisted of 26 primary items with 6 secondary branching items that were dependent on the responses to several of the primary items (e.g., if any alcohol craving was reported, participants were asked about the strength of their alcohol craving resistance). A single item regarding craving was used to inquire about craving for alcohol ("At its most severe point, how strong was your craving 
for alcohol yesterday, with 0 being not at all strong and 7 being very strong?"). Alcohol consumption was assessed via the following set of questions beginning with "Did you drink any alcohol yesterday?" Those who indicated that they did consume alcohol during the monitoring period were then asked to indicate how much of each type of alcohol they consumed first verbally (e.g., "How much beer did you drink yesterday?") and then using the key pad to enter a numeric answer (e.g., "How many standard drinks of beer did you consume yesterday? Remember, one standard drink is 12 ounces of beer.") Because many participants in our earlier study of IVR monitoring indicated having had difficulty with SDU conversions (Simpson et al., 2005), we did extensive training on SDU conversions with each participant based, in part, on their typical drinking during the baseline period.

In addition to questions regarding alcohol and drug cravings and use, we retained many of Searles et al.'s (1995) items regarding mood and general functioning and added items regarding sleep quality, level of anger, as well as six items from the PCL-C (upsetting dreams, upset due to reminders, avoiding reminders, alertness, emotionally numb, startle response, or jumpiness) that were used in our previous work on IVR daily monitoring (Simpson et al., 2005).

\section{Study Treatments}

Medications. Participants were titrated to a target dose of $4 \mathrm{mg}$ QAM, $4 \mathrm{mg}$ QPM and $8 \mathrm{mg}$ QHS (or highest tolerated dose) by the end of week 2 and then continued on this dose for an additional 4 weeks. Dosing was held at $1 \mathrm{mg}$ QHS for the first two nights to minimize the chance of first-dose syncope. The study clinician arranged participants' medication for them every 2 weeks in a mediset, to avoid confusion during the titration period.

Placebo and study medication were identically matched and were prepared by a local compounding pharmacy. Study medication underwent quality assurance testing by the VA Veterans Integrated Service Network 20 Mental Illness Research Educational and Clinical Center Pharmacology Laboratory in Boise, ID. Prazosin concentration in 2 and $4 \mathrm{mg}$ capsules measured by high performance liquid chromatography had a coefficient of variation of $<5 \%$.

Medical Management. Participants received 5 counseling visits with a study clinician over the course of the 6-week study, using the Medical Management (MM) protocol shown to be effective in reducing alcohol consumption in both the COMBINE Study (Anton et al., 2006) and a prior study of naltrexone (O’Malley et al., 2003). Designed for delivery in the primary care setting, the goal of $\mathrm{MM}$ is to facilitate recovery from AD by promoting compliance with medication through psychoeducational methods, providing support for the participant in changing his or her drinking patterns, and encouraging participation in self-help groups such as AA. Participants attended an initial session lasting 30 to 45 minutes in which the diagnosis of $\mathrm{AD}$ and its related problems as well as rationale for use of medication were addressed. Participants then received 10-minute follow-up during sessions at weeks 1, 2, 4, and 6 .

\section{Data Analysis}

All subjects were included in the analyses as randomized. Demographic, baseline, and compliance variables are presented descriptively. Differences between the placebo and prazosin groups were analyzed with the chi-square statistic for categorical variables and with student's $t$-test for continuous variables.

Multilevel mixed-effects linear regression models with random intercept and slope that included treatment group, time, and treatment group $\times$ time interaction were used to analyze changes over the 42-day study course in days of alcohol use per week and drinks per week reported via IVR daily monitoring; analyses were adjusted for days of use and total drinking over the 7 days prior to medication start based on information from the baseline Form-42, respectively. Differences in drinking days per week and drinks per week over the last three weeks of the study were analyzed in a similar manner; however, the treatment group $\times$ time interaction term was not included.

Change between baseline and week 6 in craving, as measured by the PACS, was compared across conditions using student's $t$-tests. Changes over the study course in craving and craving resistance as measured by IVR were analyzed using multilevel mixed-effects linear regression and included treatment group, time, and a treatment group $\times$ time interaction term as covariates.

Differences in adverse events and BP changes were examined using the chi-square statistic and student's $t$-test as appropriate. Analyses were conducted with Intercooled Stata version 9.2 (StataCorp LP, College Station, TX) and SPSS 13.0 for Windows (SPSS Inc., Chicago, IL). Two-sided $p$-values of $<0.05$ were considered statistically significant.

\section{RESULTS}

\section{Baseline Group Comparisons}

The two groups did not significantly differ on any demographic characteristics. The two groups also did not differ significantly over the 42-day baseline with regard to average number of days drinking [prazosin $29.6 \pm 10.4$; placebo $24.4+14.7 ; t(24)=1.0, \mathrm{~ns}$, average number of heavy days drinking [prazosin 20.1 \pm 13.2 ; placebo $19.0 \pm 14.4$; $t(24)=0.2, \mathrm{~ns}$ ], or average SDU's reported [prazosin $177.3 \pm 88.1$; placebo $208.0 \pm 149.3 ; t(24)=0.6$, ns]. Nor did the two groups differ significantly in their severity of AD as assessed via number of DSM-IV criteria present [prazosin $5.6 \pm 1.6$; placebo $5.4 \pm 1.3 ; t(24)=0.3, \mathrm{~ns}]$.

\section{Completion and Compliance Rates}

Twenty of the $24(83.3 \%)$ randomized individuals completed the trial. One individual withdrew after 3 days (assigned to placebo condition) before initiating medication or IVR monitoring; two (both assigned to prazosin) withdrew after 3 weeks because they were caring for family members and unable to attend appointments, and one was discharged from the study because he ran out of study medication and took a family member's prescription prazosin, thus compromising the randomization and the blind.

The two groups were comparable with regard to their reports of medication compliance via the daily IVR monitoring, with the prazosin group reporting having taken $92 \%$ of their study medication doses, and the placebo group reporting having taken $87 \%$ of their study medications. There were no significant differences between the two groups in the number of MM visits completed, number of vital signs visits attended, or number of lab visits attended. The study visit attendance with all 24 enrollees included was $88 \%$ of MM visits, $86 \%$ of vital sign visits, and $83 \%$ of lab visits completed (the comparable percentages for the three visit types for the 20 study completers were $91 \%, 89 \%$, and $87 \%$ respectively). The compliance rate on the IVR daily monitoring for the 23 who initiated IVR was $87.5 \%$ (for the 20 completers the compliance rate was $94.5 \%$ ). 


\section{Drinking Changes over the 42 Day Study}

In the sample as a whole, alcohol use decreased by an estimated 2.78 mean drinks per week $(95 \% \mathrm{CI}=-4.01$ to $-0.55 ; p=0.010)$ and 0.40 drinking days per week $(95 \%$ $\mathrm{CI}=-0.61$ to $-0.19 ; p<0.001)$. No differences were detected between conditions in change in mean drinks per week or mean drinking days per week over the 6-week study course. The two groups began to differ with respect to drinking days per week and drinks per week after week 3 , most likely related to the titration schedule, which does not attain a full dose until the end of week 2 . Among the 20 subjects who completed the study, the prazosin group reported fewer drinking days per week than the placebo group during the final 3 weeks of the study ( $\beta=-1.22 ; 95 \%$ CI $=-2.29$ to -0.14 ; $p=0.027)$ after adjusting for drinking days per week at baseline and week number. The average total number of drinking days during the last 3 weeks of the study for the placebo group was 5.6 (SEM 1.9) and for the prazosin group was 3.2 (SEM 1.9). Differences between the groups were not detected in mean drinks per week during the final 3 weeks.

Because other studies have found that women and men respond differently to medications for AD (Baros et al., 2008; Flannery et al., 2007; Garbutt et al., 2005; Nichol et al., 2007; O'Malley et al., 2007) in conjunction with the facts that only one woman was randomized to the control group, and only three women completed the trial, the following results are based on the 17 men who completed the study.

Six of the seven male prazosin completers reported abstinence at the final week 6 visit via both the daily IVR and the Form-42, while only four of the ten male completers in the placebo group were abstinent at week 6 (note: 1 prazosin participant and 3 control participants were abstinent during the entire 42 day trial). Controlling for drinking days per week at baseline, mixed linear regression models detected a significant difference in change over time between conditions in total drinking days per week $(\beta=-0.45 ; 95 \% \mathrm{CI}=-0.83$ to $-0.07 ; p=0.019)$. Significant differences were not detected in change over time in average drinks per week using similar models $(\beta=-3.21 ; 95 \% \mathrm{CI}=-7.31$ to $0.89 ; p=0.125)$, although there was a trend favoring the prazosin condition.

Adjusting for drinking days per week at baseline and week number, the prazosin group reported fewer drinking days per week than the placebo group during the final 3 weeks of the study $(\beta=-1.84 ; 95 \% \mathrm{CI}=-2.74$ to $-93 ; p<0.001)$ (see Fig. 1). The average total number of drinking days during the last 3 weeks of the study for the placebo group was 5.7 (SEM 1.9) and for the prazosin group was 0.9 (SEM 0.5). Likewise, after adjusting for drinks per week at baseline and week number, the prazosin group reported fewer drinks per week in the final 3 weeks of the study $(\beta=-4.59 ; 95 \% \mathrm{CI}=-8.86$ to $-0.31 ; p=0.035)$. The average total number of drinks during the last 3 weeks of the study was 20.8 (SEM 6.5) and 2.6 (SEM 1.3) for the placebo and prazosin groups, respectively. The differences between prazosin and placebo in this study are particularly notable given the robust placebo response.

\section{Craving and Craving Resistance}

Although the entire sample on average reported significantly decreased craving from baseline to the final assessment on the retrospective PACS measure [PACS: baseline $16.8 \pm 5.3$; final $8.5 \pm 4.9 ; t(19)=5.7 ; p<0.0001]$, the prazosin group did not report significantly less craving than the placebo group on this measure of craving. The same pattern of results on the PACS was found for male subjects [PACS: baseline $16.0 \pm 5.6$; final $8.1 \pm 5.3 ; t(15)=4.6$; $p<0.0001$; no between group differences].

For the sample as a whole, mean craving measured via the daily IVR system decreased by an estimated $0.93(95 \%$ $\mathrm{CI}=-1.55$ to $-0.31 ; p=0.003)$ points between weeks 1 and 6 of the study. Craving did not differ by condition. In
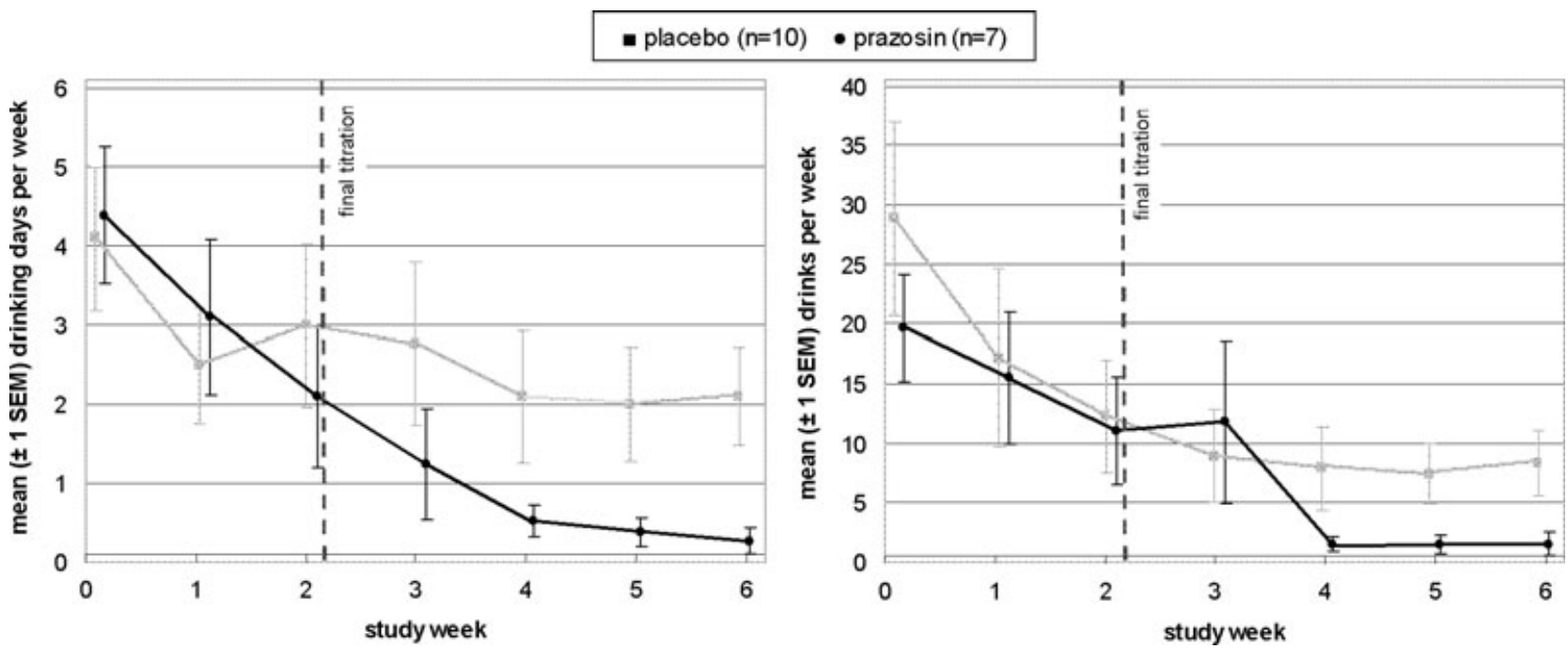

Fig. 1. Mean days drinking per week and standard drinks among male study completers by study condition. 
male subjects, craving decreased by an estimated $0.90(95 \%$ $\mathrm{CI}=-1.48$ to $-0.31 ; p=0.003)$ points. Again, no differences were detected between conditions. Across conditions, after adjusting for mean craving, no changes in mean craving resistance per week were detected for the sample as a whole or for male subjects. For the entire sample, craving resistance did not differ between conditions. For men only, the prazosin group reported greater increases over time in mean craving resistance per week after adjusting for mean craving per week $(\beta=0.36 ; 95 \%$ CI $=0.01-0.70 ; p=0.043)$.

\section{Laboratory Findings}

Among the 12 placebo treated subjects, 9 had normal aspartate amino transferase (AST) and alanine amino transferase (ALT) levels at baseline and throughout the 6-week study. Three of the placebo treated subjects had abnormal AST and ALT at baseline. One of these subjects dropped out and did not participate in follow-up testing. The other 2 subjects showed generally decreasing trends in AST and ALT but continued to show some elevations throughout the study.

Among the 12 prazosin treated subjects, 4 subjects had normal AST and ALT levels at baseline. Two of these subjects dropped out, and the remaining 2 had normal levels throughout the study. Among the remaining 8 subjects, 1 had a minimal elevation in baseline ALT which normalized during the study, 1 had elevations only in ALT which continued to be abnormal throughout the study. The other 6 subjects had baseline elevations in both enzymes. One of these subjects dropped out, and the rest maintained elevations in both enzymes throughout the course of the study.

An examination of potential correlations between alcohol consumption and liver enzymes during the course of the study showed no consistent significant relationship between these variables.

\section{Safety Findings}

There were no serious adverse events. The prazosin group reported no more negative side-effects than the placebo group. The most frequently reported side-effects were dizziness, lack of energy, and drowsiness; both the placebo group and the prazosin group reported these side-effects on average about 2.5 days of the 42 day study period. The placebo group was somewhat more apt to attribute the side-effects to the study medications. Though not statistically significant, BP changes were more evident among those in the prazosin condition, with an average drop in sitting systolic and diastolic pressure of 6.6 and $6.3 \mathrm{~mm} \mathrm{Hg}$, respectively, from the first MM visit to the final study visit (placebo condition: 1.4 decrease in sitting systolic and 3.3 decrease in sitting diastolic). There were no reports of orthostatic dizziness or other symptoms consistent with clinically meaningful orthostatic BP reduction. One individual in the prazosin group did experience one episode of syncope, which was addressed effectively by dose reduction $(1,1$, and $4 \mathrm{mg})$. Of the twenty completers, this individual was the only one who did not tolerate the full medication dosing regimen and the noncompleters all ended the study at the target dose for the titration phase they happened to be at when they left the study.

\section{Success of the Study Blind}

Completers did not correctly guess their treatment assignment at a rate greater than chance. Sixteen of the 20 completers were very confident that they were in the prazosin condition, while the remaining participants were on average somewhat certain that they were in the placebo group.

\section{DISCUSSION}

The results of the present pilot study suggest that the nonselective, $\alpha_{1}$ adrenergic antagonist, prazosin may be a useful agent for treating AD. Prazosin also appears to have been an acceptable medication to the study participants. Not only were there few reports of intolerable side-effects overall, but the two conditions did not differ significantly or clinically on this important indicator. The most common side-effects reported were dizziness, fatigue, and drowsiness. One individual reported a single incident of syncope that occurred while she had the flu, and it was managed successfully with a downward medication titration. In general clinical practice syncope is estimated to occur at a rate of $<1 \%$ of patients treated with prazosin (Carruthers, 1994). Thus, if larger trials of longer duration of prazosin for AD continue to show promise, medication side-effects are unlikely to deter its widespread use, though this is an important empirical question that remains to be tested. The fact that 19 of the 20 completers ended the study at the fully titrated dose is also a positive sign that prazosin was well tolerated and acceptable to study participants.

Although the study results strongly suggest that prazosin is a medication worthy of further study for $\mathrm{AD}$, our results regarding craving leave unanswered questions regarding mechanism(s) of action. It is possible that our single IVR craving item was insufficient to tap craving adequately, though we also failed to find any between group differences on the more traditional retrospective PACS craving measure. Another possible explanation that could be profitably explored is whether prazosin acts to reduce the reinforcing qualities of alcohol, making the experience of drinking more flat or unappealing. This could be evaluated through questions regarding how high they felt or how enjoyable alcohol was when it was consumed (Ray and Hutchison, 2007), as well as by questions regarding reasons for not drinking on days when no alcohol is consumed (e.g., "the thought of drinking wasn't appealing" or "I didn't have any desire to drink"). Future work in this area should more thoroughly assess craving.

The present study has a number of strengths to recommend it. First, the randomized double-blind placebo control design and the success of the blind allows us some confidence in 
asserting that there was a genuine medication effect untainted by demand characteristics. Second, the positive findings for prazosin among individuals with $\mathrm{AD}$ and no PTSD indicates that this medication may be a useful treatment for AD even when the common comorbidity with PTSD is not present. Third, while the use of the MM platform appears to have had salutary effects for the sample as a whole, we were thus able to control the type and amount of behavioral treatment study participants obtained during the 6-week pilot study. This allows us to infer that the active medication was useful in reducing alcohol consumption above and beyond the MM. Finally, the present study demonstrated the feasibility and the utility of incorporating a daily monitoring data collection system (IVR) into the study design. The participants were very compliant with the daily calls (see also Kranzler et al., 2004), and the frequent information allowed us to better evaluate the changes over time in drinking relative to the titration schedule. The IVR system also holds promise with regard to evaluating mediators or moderators of change at this stage of inquiry into whether prazosin is a viable pharmacological agent for treating AD. However, it should be noted that such intensive daily monitoring is unlikely to be in place in a standard clinical setting and thus, if prazosin's efficacy continues to be borne out in more comprehensive randomized trials it will be important that it is also tested in a context that better approximates standard clinical care.

The primary limitations of the study include its small sample size, the very small number of women included and their uneven distribution across the conditions, and the short 6-week timeframe of the trial. With regard to the latter issue, it will be critical that future studies of prazosin use a longer medication timeframe to assess whether more serious sideeffects develop over time and whether the promising reductions in alcohol consumption seen in our 6-week pilot results hold over a longer duration, particularly in light of the fact that risk of relapse continues throughout the first year of abstinence (Finney et al., 1996).

An additional study limitation is that we included four individuals ( 1 in the prazosin condition and 3 in the placebo condition) who were abstinent the entire 6-weeks of the study, thus making it impossible to determine whether they experienced any medication effects. Indeed, overall the present sample had levels of consumption on the lower end of the spectrum for AD (Dawson et al., 2005), which, though comparable to subjects in the COMBINE study (Anton et al., 2006) and the multisite injectable naltrexone study (Garbutt et al., 2005), does limit generalizability of these results. Another limitation is that gamma-glutamyl transferase (GGT) levels were not obtained. GGT is more sensitive to the effects of alcohol than are AST or ALT (Conigrave et al., 2003). Finally, the lack of follow-up after the medication phase is problematic as we do not know whether the positive changes in alcohol consumption for those in the prazosin condition would persist without the medication.

In light of the present findings, future studies of prazosin involving larger samples of individuals with more severe AD studied over longer timeframes are warranted. Careful consideration of potential moderators and mediators assessed daily would be extremely helpful in furthering our understanding of both how prazosin works functionally and could provide insight into the NE systems involving reinforcement (Ventura et al., 2005) and stress responsivity. Also, the inclusion of sufficient numbers of women to allow analyses regarding gender similarities and differences would be critical to more fully understanding for whom prazosin is useful. In any event, the potential promise of a pharmacotherapy for $\mathrm{AD}$ with a completely different mechanism of action from existing medications represents an encouraging development. Thus, prazosin may be an agent for individuals who cannot tolerate or have contraindications for other medications for AD. Finally, should it be found to be effective for treating $\mathrm{AD}$ in larger trials, further research could investigate whether prazosin in combination with agents that targets different neurobiological systems has a synergistic effect that leads to improved efficacy.

\section{ACKNOWLEDGMENTS}

The funding for this study was provided by the VA Puget Sound Health Care System, Seattle, Washington Center of Excellence in Substance Abuse Treatment and Education and by a grant from the University of Washington's Alcohol and Drug Abuse Institute. The authors wish to thank the following individuals for the invaluable assistance with this project: Lynne Hyerle, M.A., Norah Sullivan, ARNP, David Hoff, PA, Craig Jaffe, MD, and Kathleen Ang Lee, MD.

\section{REFERENCES}

Anton RF, O'Malley SS, Ciraulo DA, Cisler RA, Couper D, Donovan DM, Gastfriend DR, Hosking JD, Johnson BA, LoCastro JS, Longabaugh R, Mason BJ, Mattson ME, Miller WR, Pettinati HM, Randall CL, Swift R, Weiss RD, Williams LD, Zweben A (2006) Combined pharmacotherapies and behavioral interventions for alcohol dependence: the COMBINE study: a randomized controlled trial. JAMA 295:2003-2017.

Anton RF, Swift RM (2003) Current pharmacotherapies of alcoholism: a U.S. perspective. Am J Addict 12(Suppl. 1):S53-S68.

Aston-Jones G, Cohen JD (2005) An integrative theory of locus coeruleusnorepinephrine function: adaptive gain and optimal performance. Annu Rev Neurosci 28:403-450.

Baros AM, Latham PK, Anton RF (2008) Naltrexone and cognitive behavioral therapy for the treatment of alcohol dependence: do sex differences exist? Alcohol Clin Exp Res 32:771-776.

Begleiter H, Porjesz B (1999) What is inherited in the predisposition toward alcoholism? A proposed model. Alcohol Clin Exp Res 23:1125-1135.

Carruthers SG (1994) Adverse effects of alpha 1-adrenergic blocking drugs. Drug Saf 11:12-20.

Chester JA, Blose AM, Froehlich JC (2004) Acoustic startle reactivity during acute alcohol withdrawal in rats that differ in genetic predisposition toward alcohol drinking: effect of stimulus characteristics. Alcohol Clin Exp Res 28:677-687.

Chick J, Gough K, Falkowski W, Kershaw P, Hore B, Mehta B, Ritson B, Ropner R, Torley D (1992) Disulfiram treatment of alcoholism. Br J Psychiatry 161:84-89.

Conigrave KM, Davies P, Haber P, Whitfield JB (2003) Traditional markers of excessive alcohol use. Addiction 98(Suppl. 2):31-43. 
Darracq L, Blanc G, Glowinski J, Tassin JP (1998) Importance of the noradrenaline-dopamine coupling in the locomotor activating effects of D-amphetamine. J Neurosci 18:2729-2739.

Dawson DA, Grant BF, Li TK (2005) Quantifying the risks associated with exceeding recommended drinking limits. Alcohol Clin Exp Res 29:902-908.

Dobie DJ, Kivlahan DR, Maynard C, Bush KR, McFall M, Epler AJ, Bradley KA (2002) Screening for post-traumatic stress disorder in female Veteran's Affairs patients: validation of the PTSD checklist. Gen Hosp Psychiatry 24:367-374.

Ehrenreich H, Schuck J, Stender N, Pilz J, Gefeller O, Schilling L, Poser W, Kaw S (1997) Endocrine and hemodynamic effects of stress versus systemic $\mathrm{CRF}$ in alcoholics during early and medium term abstinence. Alcohol Clin Exp Res 21:1285-1293.

Finney JW, Hahn AC, Moos RH (1996) The effectiveness of inpatient and outpatient treatment for alcohol abuse: the need to focus on mediators and moderators of setting effects. Addiction 91:1773-1796; discussion 1803-20.

First MB, Spitzer RL, Gibbon M (1995) Structured Interview for DSM-IV Axis I Disorders. State Psychiatric Institute, Biometrics Research, New York, NY.

Flannery B, Fishbein D, Krupitsky E, Langevin D, Verbitskaya E, Bland C, Bolla K, Egorova V, Bushara N, Tsoy M, Zvartau E (2007) Gender differences in neurocognitive functioning among alcohol-dependent Russian patients. Alcohol Clin Exp Res 31:745-754.

Flannery BA, Volpicelli JR, Pettinati HM (1999) Psychometric properties of the Penn Alcohol Craving Scale. Alcohol Clin Exp Res 23:1289-1295.

Fuller RK, Branchey L, Brightwell DR, Derman RM, Emrick CD, Iber FL, James KE, Lacoursiere RB, Lee KK, Lowenstam I, Maany I, Neiderhiser D, Nocks JJ, Shaw S (1986) Disulfiram treatment of alcoholism. A Veterans Administration cooperative study. JAMA 256:1449-1455.

Fuller RK, Roth HP (1979) Disulfiram for the treatment of alcoholism. An evaluation in 128 men. Ann Intern Med 90:901-904.

Fuller R, Roth H, Long S (1983) Compliance with disulfiram treatment of alcoholism. J Chronic Dis 36:161-170.

Garbutt JC, Kranzler HR, O’Malley SS, Gastfriend DR, Pettinati HM, Silverman BL, Loewy JW, Ehrich EW (2005) Efficacy and tolerability of long-acting injectable naltrexone for alcohol dependence: a randomized controlled trial. JAMA 293:1617-1625.

Garbutt JC, West SL, Carey TS, Lohr KN, Crews FT (1999) Pharmacological treatment of alcohol dependence: a review of the evidence. JAMA 281:1318-1325.

Gianoulakis C (2004) Endogenous opioids and addiction to alcohol and other drugs of abuse. Curr Topics Med Chem 4:39-50.

Gonzales RA, Job MO, Doyon WM (2004) The role of mesolimbic dopamine in the development and maintenance of ethanol reinforcement. Pharmacol Ther 103:121-146.

Heath AC, Bucholz KK, Madden PA, Dinwiddie SH, Slutske WS, Bierut LJ, Statham DJ, Dunne MP, Whitfield JB, Martin NG (1997) Genetic and environmental contributions to alcohol dependence risk in a national twin sample: consistency of findings in women and men. Psychol Med 27:13811396.

Hieble JP, Ruffolo RR Jr (1996) The use of alpha-adrenoceptor antagonists in the pharmacological management of benign prostatic hypertrophy: an overview. Pharmacol Res 33:145-160.

Johnson BA, Rosenthal N, Capece JA, Wiegand F, Mao L, Beyers K, McKay A, Ait-Daoud N, Anton RF, Ciraulo DA, Kranzler HR, Mann K, O'Malley SS, Swift RM (2007) Topiramate for treating alcohol dependence: a randomized controlled trial. JAMA 298:1641-1651.

Kendler KS, Neale MC, Heath AC, Kessler RC, Eaves LJ (1994) A twinfamily study of alcoholism in women. Am J Psychiatry 151:707-715.

Koob GF (1999) Corticotropin-releasing factor, norepinephrine, and stress. Biol Psychiatry 46:1167-1180.

Koob GF (2004) A role for GABA mechanisms in the motivational effects of alcohol. Biochem Pharmacol 68:1515-1525.

Kranzler HR, Abu-Hasaballah K, Tennen H, Feinn R, Young K (2004) Using daily interactive voice response technology to measure drinking and related behaviors in a pharmacotherapy study. Alcohol Clin Exp Res 28:10601064.
Krystal JH, Staley J, Mason G, Petrakis IL, Kaufman J, Harris RA, Gelernter J, Lappalainen J (2006) Gamma-aminobutyric acid type A receptors and alcoholism: intoxication, dependence, vulnerability, and treatment. Arch Gen Psychiatry 63:957-968.

Krystal JH, Webb E, Grillon C, Cooney N, Casal L, Morgan CA 3rd, Southwick SM, Davis M, Charney DS (1997) Evidence of acoustic startle hyperreflexia in recently detoxified early onset male alcoholics: modulation by yohimbine and $\mathrm{m}$-chlorophenylpiperazine (mCPP). Psychopharmacology (Berl) 131:207-215.

Lund-Johansen P, Hjermann I, Iversen BM, Thaulow E (1993) Selective alpha-1 inhibitors: first- or second-line antihypertensive agents? Cardiology 83:150-159.

Mann K, Lehert P, Morgan MY (2004) The efficacy of acamprosate in the maintenance of abstinence in alcohol-dependent individuals: results of a meta-analysis. Alcohol Clin Exp Res 28:51-63.

Mason BJ, Goodman AM, Chabac S, Lehert P (2006) Effect of oral acamprosate on abstinence in patients with alcohol dependence in a double-blind, placebo-controlled trial: the role of patient motivation. J Psychiatr Res 40:383-393.

Mason BJ, Kocsis JH (1991) Desipramine treatment of alcoholism. Psychopharmacol Bull 27:155-161.

Mason BJ, Kocsis JH, Ritvo EC, Cutler RB (1996) A double-blind, placebocontrolled trial of desipramine for primary alcohol dependence stratified on the presence or absence of major depression. JAMA 275:761-767.

Menkes DB, Baraban JM, Aghajanian GK (1981) Prazosin selectively antagonizes neuronal responses mediated by alphal-adrenoceptors in brain. Naunyn Schmiedebergs Arch Pharmacol 317:273-275.

Miller WR, Del Boca FK (1994) Measurement of drinking behavior using the Form 90 family of instruments. J Stud Alcohol Suppl 12:112-118.

Nagy J (2004) Renaissance of NMDA receptor antagonists: do they have a role in the pharmacotherapy for alcoholism? IDrugs 7:339-350.

Nichol PE, Krueger RF, Iacono WG (2007) Investigating gender differences in alcohol problems: a latent trait modeling approach. Alcohol Clin Exp Res 31:783-794.

O'Malley SS, Rounsaville BJ, Farren C, Namkoong K, Wu R, Robinson J, O'Connor PG (2003) Initial and maintenance naltrexone treatment for alcohol dependence using primary care vs specialty care: a nested sequence of 3 randomized trials. Arch Intern Med 163:1695-1704.

O'Malley SS, Sinha R, Grilo CM, Capone C, Farren CK, McKee SA, Rounsaville BJ, Wu R (2007) Naltrexone and cognitive behavioral coping skills therapy for the treatment of alcohol drinking and eating disorder features in alcohol-dependent women: a randomized controlled trial. Alcohol Clin Exp Res 31:625-634.

Orr SP, Lasko NB, Shalev AY, Pitman RK (1995) Physiologic responses to loud tones in Vietnam veterans with posttraumatic stress disorder. J Abnorm Psychol 104:75-82.

Oswald LM, Wand GS (2004) Opioids and alcoholism. Physiol Behav 81:339358.

Patkar AA, Gopalakrishnan R, Naik PC, Murray HW, Vergare MJ, Marsden CA (2003) Changes in plasma noradrenaline and serotonin levels and craving during alcohol withdrawal. Alcohol Alcohol 38:224-231.

Patkar AA, Marsden CA, Naik PC, Kendall DA, Gopalakrishnan R, Vergare MJ, Weinstein SP (2004) Differences in peripheral noradrenergic function among actively drinking and abstinent alcohol-dependent individuals. Am J Addict 13:225-235.

Rasmussen DD, Alexander LL, Raskind MA, Froehlich JC (2008) The alpha1 adrenergic receptor antagonist, prazosin, reduces alcohol drinking in alcohol-preferring (P) rats. Alcohol Clin Exp Res (In press).

Rasmussen DD, Burke B, Crites NJ (2005) Chronic daily ethanol and withdrawal: melatonin treatment reverses persistently increased acoustic startle response during abstinence. Alcohol Clin Exp Res 29:16A.

Ray LA, Hutchison KE (2007) Effects of naltrexone on alcohol sensitivity and genetic moderators of medication response: a double-blind placebocontrolled study. Arch Gen Psychiatry 64:1069-1077.

Searles JS, Helzer JE, Walter DE (2000) Comparison of drinking patterns measured by daily reports and timeline follow back. Psychol Addict Behav 14:277-286. 
Searles JS, Perrine MW, Mundt JC, Helzer JE (1995) Self-report of drinking using touch-tone telephone: extending the limits of reliable daily contact. J Stud Alcohol 56:375-382.

Simpson TL, Kivlahan DR, Bush KR, McFall ME (2005) Telephone selfmonitoring among alcohol use disorder patients in early recovery: a randomized study of feasibility and measurement reactivity. Drug Alcohol Depend 79:241-250.

Stevens DR, McCarley RW, Greene RW (1994) The mechanism of noradrenergic alpha 1 excitatory modulation of pontine reticular formation neurons. J Neurosci 14:6481-6487.

Swift RM (1999) Drug therapy for alcohol dependence. N Engl J Med 340:1482-1490

Tjurmina OA, Goldstein DS, Palkovits M, Kopin IJ (1999) Alpha2-adrenoceptor-mediated restraint of norepinephrine synthesis, release, and turnover during immobilization in rats. Brain Res 826:243-252.

Tupala E, Tiihonen J (2004) Dopamine and alcoholism: neurobiological basis of ethanol abuse. Progress Neuro Psychopharmacol Biol Psychiatry 28:1221-1247.
Ventura R, Alcaro A, Puglisi-Allegra S (2005) Prefrontal cortical norepinephrine release is critical for morphine-induced reward, reinstatement and dopamine release in the nucleus accumbens. Cereb Cortex 15:1877-1886.

Walker BM, Rasmussen DD, Raskind MA, Koob GF (2008) $\alpha^{1}$-noradrenergic receptor antagonism blocks dependence-induced increases in responding for alcohol. Alcohol 42:91-97.

Weathers FW, Litz BT, Heerman DS, Huska JA, Keane TM (1993) The PTSD Checklist (PCL): Reliability, Validity, and Diagnostic Utility. Abstract, 9th annual meeting of the International Society for Traumatic Stress Studies, San Antonio, TX.

Westfall TC, Westfall DP (2006) Neurotransmission: the automatic and somatic motor nervous system, in Goodman \& Gilman's The Pharmacological Basis of Therapeutics (Brunton L, Lazo J, Parker K eds), pp 237-295. McGraw-Hill, New York.

Woodward JJ, Ron D, Winder D, Roberto M (2006) From blue states to up states: a regional view of NMDA-ethanol interactions. Alcohol Clin Exp Res 30:359-367. 